\title{
The velocity-scalar cross spectrum of stretched spiral vortices
}

\author{
P. A. O'Gorman ${ }^{\text {a) }}$ and D. I. Pullin \\ Graduate Aeronautical Laboratories 105-50, California Institute of Technology, Pasadena, California 91125
}

(Received 2 July 2002; accepted 17 October 2002; published 19 December 2002)

\begin{abstract}
The stretched-spiral vortex model is used to calculate the velocity-scalar cross spectrum for homogeneous, isotropic turbulence in the presence of a mean scalar gradient. The only nonzero component of the cospectrum is that contributed by the velocity component in the direction of the imposed scalar gradient while the quadrature spectrum is identically zero, in agreement with experiment. For the velocity field provided by the stretched-spiral vortex, the velocity-scalar spectrum can be divided into two additive components contributed by the velocity components along the vortex axis, and in the plane normal to this axis, respectively. For the axial velocity field, a new exact solution of the scalar convection-diffusion equation is found exhibiting scalar variation in the direction of the vortex tube axis. An asymptotic expression was found for the cospectrum contributed by this solution and the axial velocity, with the leading order term showing a $k^{-5 / 3}$ range. This term is produced by the winding of the initial axial velocity field by the axisymmetric vortex core. The next order term gives a $k^{-7 / 3}$ range, and arises from the lowest order effect of the nonaxisymmetric vorticity on the evolution of the axial velocity. Its coefficient can be of either sign or zero depending on the initial conditions. The contribution to the cospectrum from the velocity in the plane of the vortex is also calculated, but no universal high wave number asymptotic form is found. The integrals are evaluated numerically and it is found that the resulting cospectrum does not remain of one sign. Its form depends on the choice of the vortex core velocity profile and time cutoff in the spectral integrals. The one-dimensional cospectrum contributed by the axial velocity is compared with the experimental data of Mydlarski and Warhaft [J. Fluid Mech. 358, 135-175 (1998)]. (C) 2003 American Institute of Physics. [DOI: 10.1063/1.1527916]
\end{abstract}

\section{INTRODUCTION}

The problem of turbulent scalar mixing shares many of the features of the classical turbulence problem, and has important applications in areas such as turbulent combustion and dispersion in both geophysical and environmental flows. It is of interest to apply methods that have been shown to provide quantitative results for the energy spectrum to the problems of scalar spectra and cross spectra in turbulent flows, one example being the recent use of the stretched spiral-vortex model to calculate the scalar spectrum ${ }^{1}$ for homogeneous but nonisotropic turbulence. As is noted below, the real part of the velocity-scalar cross spectrum gives the distribution of the scalar flux across scales, and thus is interesting for problems in turbulent heat transfer. Also, because a mean scalar flux can only occur as a result of anisotropy, it is interesting to know how quickly the cross spectrum decays with increasing wave number. If, as is thought, the cross spectrum decays faster than the scalar or energy spectra, then this is a measure of the approach to isotropy at the smaller scales. In addition, because the total scalar flux represents transport in the scalar advection-diffusion equation, knowledge of the cross-spectral properties of vortex-models is expected to be useful in their application to the building of subgrid scalar-flux and mixing models for use in large-eddy simulation. $^{2}$

\footnotetext{
a) Author to whom correspondence should be addressed. Mail Stop 205-45, Caltech, Pasadena, CA 91125; electronic mail: pog@caltech.edu
}

If we define the velocity-scalar correlation by

$$
R_{u_{i} c}(\mathbf{r})=\overline{u_{i}(\mathbf{x}) c(\mathbf{x}+\mathbf{r})},
$$

then the one-dimensional velocity-scalar cross spectrum is given by

$$
F_{u_{i} c}^{1 d}\left(k_{3}\right)=\frac{1}{2 \pi} \int_{-\infty}^{\infty} R_{u_{i} c}\left(0,0, r_{3}\right) e^{-i k_{3} r_{3}} d r_{3} .
$$

In general the cross spectrum may be complex and can be split into real and imaginary parts as

$$
2 F_{u_{i} c}^{1 d}\left(k_{3}\right)=C_{u_{i} c}^{1 d}\left(k_{3}\right)-i Q_{u_{i} c}^{1 d}\left(k_{3}\right),
$$

where $C_{u_{i} c}^{1 d}\left(k_{3}\right)$ is the cospectrum and $Q_{u_{i} c}^{1 d}\left(k_{3}\right)$ is the quadrature spectrum. ${ }^{3}$ The quadrature spectrum is related to phase differences between the Fourier components of the scalar and the velocity fields. In Appendix A the quadrature spectrum is shown to be zero for the case of isotropic turbulence and a mean scalar gradient. The integral of the cospectrum over all wave numbers is equal to the scalar flux,

$$
\overline{u_{i} c}=\int_{0}^{\infty} C_{u_{i} c}^{1 d}\left(k_{3}\right) d k_{3} \text {. }
$$

While considering the effect of buoyancy on the energy spectrum, Lumley ${ }^{4}$ used a similarity hypothesis to predict the shell-summed cospectrum of the velocity and potential temperature in the inertial-convective range. If the gravitational 
force is set to zero, then the absolute and potential temperatures are the same, and Lumley's Eq. (12) for the cospectrum simplifies to

$$
C_{u_{1} c}(k) \sim \mu \epsilon^{1 / 3} k^{-7 / 3},
$$

where $\epsilon$ is the mean energy dissipation and $\mu$ is the mean scalar (temperature) gradient in the " 1 "-direction. We are assuming the flow is such that temperature is approximately a passive scalar, and the turbulent velocity field is isotropic. Note that Eq. (5) follows from dimensional analysis if the cospectrum depends only on $k, \epsilon$ and $\mu$.

Mydlarski and Warhaft ${ }^{5}$ studied the velocity-temperature cross spectrum in a wind tunnel for Taylor Reynolds numbers as high as 582. Fluctuations in the passive temperature field were generated by imposing a linear mean temperature gradient across the tunnel. The resulting cross spectrum was noisier than the energy or scalar spectra, and this was explained by noting that no mathematical limitation keeps the spectrum either positive or negative. Nonetheless their results indicate that the quadrature spectrum is close to zero, while the cospectrum exhibited a $k^{-2}$ range at large $R_{\lambda}$.

The stretched spiral-vortex model of turbulence, introduced by Lundgren, ${ }^{6}$ uses an ensemble of vortex tubes to model the fine scales of turbulence. The vortex tubes do not interact except in that they are stretched on average by the surrounding flow. The vortex tubes are assumed to be straight, with no dependence of the velocity field on the coordinate parallel to the tube axis. In each tube the vorticity is evolved by the Navier-Stokes equations and the scalar is evolved by the convection-diffusion equation. The axial vorticity, the axial velocity and the scalar are each wound up into spirals by the differential rotation of the cores of the vortices. Average flow statistics are calculated by performing an average over time and space. This model gives good results for energy ${ }^{6}$ and scalar spectra ${ }^{1}$ individually. By also performing an average over vortex orientation the model was used to calculate vorticity and velocity-derivative moments, ${ }^{7}$ as well as one dimensional spectra. ${ }^{8}$

There have been few attempts to calculate the velocityscalar cross spectrum; see for example the EDQNM calculations of Herr, Wang and Collins. ${ }^{9}$ In this paper we propose to calculate the cross spectrum using the stretched spiral-vortex model. To model the experiment of Mydlarski and Warhaft, a mean scalar gradient is imposed and an isotropic turbulent velocity field is assumed, with the vortex tubes oriented with equal probability in all directions. In Sec. II asymptotic expressions for the evolution of the velocity and scalar fields in the vortex tube are described. A new feature of the present analysis is that the scalar can now show variation parallel to the vortex axis, and so its evolution is influenced by the axial velocity. This is important because the scalar and the axial velocity evolve in a similar way, unlike the scalar and a given planar component of the velocity, leading to an important contribution to the velocity-scalar correlation. In Sec. III expressions are derived for the cross spectrum contributed by the axial velocity and also the velocity in the plane of the vortex. It is found that the only nonzero cross spectrum is that of the scalar and the velocity in the direction of the scalar gradient. In Sec. IV the expressions for the cross spectrum are evaluated for a particular choice of initial conditions. The contribution from the axial velocity is evaluated using its asymptotic form for high wave number, but the contribution from the velocity in the plane of the vortex can only be evaluated numerically.

\section{EVOLUTION OF THE SCALAR AND THE VELOCITY IN A STRETCHED VORTEX TUBE}

We wish to find the evolution of the velocity and scalar fields in a vortex tube embedded in a background linear velocity field. For convenience let $x_{i}$ now be vortex fixed axes where $x_{3}$ is aligned with the vortex tube axis, and let $x_{i}^{\prime}$ be the laboratory coordinates. The velocity field of the vortex tube is assumed independent of the axial coordinate $x_{3}$, but may have a component in the direction of the vortex axis. The scalar is a function of all three spatial coordinates. The following analysis generalizes that of Pullin and Lundgren, ${ }^{1}$ hereafter referred to as PL, by letting the scalar have an $x_{3}$ dependence. The effect of this change on the scalar spectrum is discussed in Appendix B.

The Navier-Stokes equations for the velocity $v_{i}$ and the vorticity $\omega_{i}$ are

$$
\begin{aligned}
& \frac{\partial v_{i}}{\partial t}+v_{j} \frac{\partial v_{i}}{\partial x_{j}}=-\frac{\partial P}{\partial x_{i}}+\nu \nabla^{2} v_{i}, \\
& \frac{\partial \omega_{i}}{\partial t}+v_{j} \frac{\partial \omega_{i}}{\partial x_{j}}=\omega_{j} \frac{\partial v_{i}}{\partial x_{j}}+\nu \nabla^{2} \omega_{i},
\end{aligned}
$$

and the convection-diffusion equation for the scalar $c\left(x_{1}, x_{2}, x_{3}, t\right)$ is

$$
\frac{\partial c}{\partial t}+v_{j} \frac{\partial c}{\partial x_{j}}=D \nabla^{2} c
$$

where $P$ is the pressure-density ratio, $\nu$ is the viscosity and $D$ is the scalar diffusivity. The velocity field is decomposed as

$$
v_{i}=u_{i}\left(x_{1}, x_{2}, t\right)+a_{i}(t) x_{i}
$$

with $a_{1}+a_{2}+a_{3}=0$ and $a_{3}>a_{2}>a_{1}$. Summation over $i$ is not implied. If the support of the vorticity is compact in a domain surrounding $x_{1}=x_{2}=0$ then the velocity can be expressed in terms of a vector potential $\psi_{i}\left(x_{1}, x_{2}, t\right)$ as

$$
u_{1}=\frac{\partial \psi_{3}}{\partial x_{2}}, \quad u_{2}=-\frac{\partial \psi_{3}}{\partial x_{1}}, \quad u_{3}=\frac{\partial \psi_{2}}{\partial x_{1}}-\frac{\partial \psi_{1}}{\partial x_{2}} .
$$

Now choose the gauge of $\psi_{i}$ so that $\partial \psi_{i} / \partial x_{i}=0$. Then

$$
\omega_{i}\left(x_{1}, x_{2}, t\right)=-\nabla_{2}^{2} \psi_{i}, \quad \nabla_{2}^{2} \equiv \frac{\partial^{2}}{\partial x_{1}^{2}}+\frac{\partial^{2}}{\partial x_{2}^{2}} .
$$

We also define a reduced pressure $P^{*}$ as follows:

$$
P^{*}\left(x_{1}, x_{2}, t\right)=P+\frac{1}{2}\left(a_{1}^{2} x_{1}^{2}+a_{2}^{2} x_{2}^{2}+a_{3}^{2} x_{3}^{2}\right) .
$$

Then we have the following equations for $\omega_{3}, u_{3}$ and $c$ : 


$$
\begin{aligned}
\frac{\partial \omega_{3}}{\partial t} & +\left(a_{1} x_{1}+\frac{\partial \psi_{3}}{\partial x_{2}}\right) \frac{\partial \omega_{3}}{\partial x_{1}}+\left(a_{2} x_{2}-\frac{\partial \psi_{3}}{\partial x_{1}}\right) \frac{\partial \omega_{3}}{\partial x_{2}}+a_{3} \omega_{3} \\
& =\nu \nabla_{2}^{2} \omega_{3} \\
\frac{\partial u_{3}}{\partial t} & +\left(a_{1} x_{1}+\frac{\partial \psi_{3}}{\partial x_{2}}\right) \frac{\partial u_{3}}{\partial x_{1}}+\left(a_{2} x_{2}-\frac{\partial \psi_{3}}{\partial x_{1}}\right) \frac{\partial u_{3}}{\partial x_{2}}+a_{3} u_{3} \\
& =\nu \nabla_{2}^{2} u_{3}, \\
\frac{\partial c}{\partial t}+ & \left(a_{1} x_{1}+\frac{\partial \psi_{3}}{\partial x_{2}}\right) \frac{\partial c}{\partial x_{1}}+\left(a_{2} x_{2}-\frac{\partial \psi_{3}}{\partial x_{1}}\right) \frac{\partial c}{\partial x_{2}} \\
& =-\left(a_{3} x_{3}+u_{3}\right) \frac{\partial c}{\partial x_{3}}+D \nabla^{2} c .
\end{aligned}
$$

It can be seen that Eqs. (13) and (11) $(i=3)$ are sufficient to determine $\omega_{3}\left(x_{1}, x_{2}, t\right)$ and $\psi_{3}\left(x_{1}, x_{2}, t\right)$. Once these are solved Eq. (14) can be solved for $u_{3}\left(x_{1}, x_{2}, t\right)$, and finally Eq. (15) can be solved for $c\left(x_{1}, x_{2}, x_{3}, t\right)$.

We wish to consider the case where there is a mean gradient, $\mu$, in the scalar in the lab frame. We will assume the mean gradient is along the $x_{1}^{\prime}$ axis, where $x_{i}^{\prime}$ are lab coordinates. The scalar field at time zero may then be decomposed as the sum of the linear gradient fixed in the lab frame, and a fluctuation $c^{\prime}(\mathbf{x}, 0)=c^{\prime}\left(x_{1}, x_{2}, x_{3}, 0\right)$,

$$
\begin{aligned}
c(t=0) & =\mu x_{1}^{\prime}+c^{\prime}(\mathbf{x}, 0) \\
& =\mu\left(E_{11} x_{1}+E_{21} x_{2}+E_{31} x_{3}\right)+c^{\prime}(\mathbf{x}, 0),
\end{aligned}
$$

where $E_{i j}(\alpha, \beta, \gamma)$ is a rotation matrix describing a rotation from the $x_{i}^{\prime}$ axes to the $x_{i}$ axes, such that $x_{j}^{\prime}=E_{i j} x_{i}$, and where $(\alpha, \beta, \gamma)$ are the corresponding Euler angles. ${ }^{8}$ Noting that the equation governing the scalar is linear, we decompose the scalar field at time $t$ as

$c(\mathbf{x}, t)=E_{11} c_{1}(\mathbf{x}, t)+E_{21} c_{2}(\mathbf{x}, t)+E_{31} c_{3}(\mathbf{x}, t)+c^{\prime}(\mathbf{x}, t)$,

where

$$
c_{i}(\mathbf{x}, 0)=\mu x_{i},
$$

and each of $c_{1}, c_{2}, c_{3}, c^{\prime}$ solve Eq. (15). We now further specialize to the case of a time independent axisymmetric strain field, $a_{1}=a_{2}=-a / 2, a_{3}=a, a>0$, and also set the Schmidt number equal to unity, $\mathrm{Sc}=\nu / D=1$.

\section{A. Solution for $c_{3}$ in terms of $u_{3}$}

We begin by defining the material derivative $D / D t$ $=\partial / \partial t+v_{i} \partial / \partial x_{i}$ and temporarily set $\nu=D=0$. Then we can rewrite Eq. (15) for $c_{3}$ as $D c_{3} / D t=0$, and it is clear that $c_{3}$ is conserved along paths $X_{i}(t)$ that satisfy $d X_{i} / d t=v_{i}$. Therefore, using the initial condition (19) we have that $c_{3}(\mathbf{x}, t)=\mu X_{3}(0)$ where $X_{i}(t)=x_{i}$. We can also rewrite Eq. (14) as $D u_{3} / D t=-a u_{3}$, so that $u_{3}(\mathbf{X}, t)=\exp (-a t) u_{3_{0}}$ where $u_{3_{0}}$ is a constant, and

$$
\frac{d X_{3}(t)}{d t}=a X_{3}(t)+e^{-a t} u_{30} .
$$

This equation can be solved to give

$$
X_{3}(0)=X_{3}(t) e^{-a t}-u_{3}\left(x_{1}, x_{2}, t\right) \frac{1}{a} \sinh (a t),
$$

so that

$$
c_{3}(\mathbf{x}, t)=\mu\left(e^{-a t} x_{3}-u_{3}\left(x_{1}, x_{2}, t\right) \frac{1}{a} \sinh (a t)\right) .
$$

It is easily verified by direct substitution that this solution (22) for $c_{3}$ is also valid in the case when $\nu \neq 0$ if $S c$ $=\nu / D=1$. The Schmidt number was 0.71 in the experiments of Mydlarski and Warhaft. ${ }^{5}$ To simplify the analysis, we subsequently assume $\mathrm{Sc}=1$.

\section{B. Solutions for $c_{1}, c_{2}$ and the axial velocity and vorticity}

Both $c_{1}$ and $c_{2}$ have no $x_{3}$ dependence initially, and so from Eq. (15) it is clear that they will be independent of $x_{3}$ at later times. We are thus motivated to study solutions to Eq. (15) when there is no $x_{3}$ dependence,

$$
\frac{\partial c}{\partial t}+\left(a_{1} x_{1}+\frac{\partial \psi_{3}}{\partial x_{2}}\right) \frac{\partial c}{\partial x_{1}}+\left(a_{2} x_{2}-\frac{\partial \psi_{3}}{\partial x_{1}}\right) \frac{\partial c}{\partial x_{2}}=D \nabla^{2} c
$$

It is convenient to work in polar coordinates $(r, \theta)$ with $x_{1}$ $=r \cos \theta, x_{2}=r \sin \theta$, and introduce the transformation ${ }^{6}$

$$
\begin{aligned}
& S(t)=e^{a t}, \\
& \rho=S(t)^{1 / 2} r, \\
& \tau=\frac{1}{a}(S(t)-1), \\
& \psi_{3}(r, \theta, t)=\widetilde{\psi}_{3}(\rho, \theta, \tau), \\
& \omega_{3}(r, \theta, t)=S(t) \widetilde{\omega}_{3}(\rho, \theta, \tau), \\
& u_{3}(r, \theta, t)=S(t)^{-1} U_{3}(\rho, \theta, \tau), \\
& c(r, \theta, t)=C(\rho, \theta, \tau) .
\end{aligned}
$$

Equations (14) and (23) can then be expressed in essentially the same form

$$
\frac{\partial \phi}{\partial t}+\frac{1}{\rho}\left(\frac{\partial \widetilde{\psi}_{3}}{\partial \theta} \frac{\partial \phi}{\partial \rho}-\frac{\partial \tilde{\psi}_{3}}{\partial \rho} \frac{\partial \phi}{\partial \theta}\right)=\kappa \nabla_{2}^{2} \phi,
$$

where $(\phi, \kappa)$ are either $\left(U_{3}, \nu\right)$ or $(C, D)$. Approximate solutions for $\widetilde{\omega}_{3}, \widetilde{\psi}_{3}, U_{3}$ and $C$ can be found using a two time analysis. ${ }^{1}$ These solutions are asymptotically correct for large $\tau$. The solution for the axial vorticity and the stream function takes the form

$$
\begin{array}{ll}
\widetilde{\omega}_{3}=\sum_{-\infty}^{\infty} \omega_{n}(\rho, \tau) \exp (\text { in } \theta), & \omega_{-n}=\omega_{n}^{*}, \\
\widetilde{\psi}_{3}=\sum_{-\infty}^{\infty} \psi_{n}(\rho, \tau) \exp (\text { in } \theta), \quad \psi_{-n}=\psi_{n}^{*},
\end{array}
$$

where the Fourier coefficients, for $n \neq 0$, are

$$
\omega_{n}(\rho, \tau)=f_{n}(\rho) \exp \left(-i n \Omega(\rho) \tau-\nu n^{2} \Lambda^{2} \tau^{3} / 3\right),
$$




$$
\psi_{n}(\rho, \tau)=\tau^{-2} h_{n}(\rho) \exp \left(-i n \Omega(\rho) \tau-\nu n^{2} \Lambda^{2} \tau^{3} / 3\right),
$$

with

$$
h_{n}(\rho)=\frac{f_{n}(\rho)}{n^{2} \Lambda^{2}}, \quad \Lambda(\rho)=\frac{d \Omega(\rho)}{d \rho} .
$$

The $\theta$ averaged angular velocity $\Omega(\rho)$ is related to the zeroth harmonic of the vorticity and to $\psi_{0}$ by

$$
\omega_{0}(\rho)=\frac{1}{\rho} \frac{\partial\left(\rho^{2} \Omega\right)}{\partial \rho}, \quad \Omega(\rho)=-\frac{1}{\rho} \frac{\partial \psi_{0}}{\partial \rho} .
$$

It should be noted that we assume $\Lambda<0$ in order for the expansion in large $\tau$ to be valid. The expressions for $\omega_{n}$ are valid to $O(1)$ for $n \neq 0$. However $\omega_{0}$ (and hence $\Omega$ ) is constant in time to within terms of order $\tau^{-2}$. The functions $f_{n}(\rho)$ may be viewed as initial conditions that define a spiral vortex structure although the solution is of course only valid for large $\tau$. The above solution for the axial vorticity essentially describes the winding of the nonaxisymmetric part of the vorticity field by the axisymmetric part (the core). The solution for the stream function may be rewritten in the form

$$
\begin{aligned}
& \widetilde{\psi}_{3}=\widetilde{\psi}^{(0)}+\tau^{-2} \widetilde{\psi}^{(2)}, \\
& \widetilde{\psi}^{(2)}=\sum_{-\infty, n \neq 0}^{\infty} \widetilde{\psi}_{n}^{(2)} \exp (i n(\theta-\Omega \tau)), \\
& \widetilde{\psi}_{n}^{(2)}=h_{n}(\rho) \exp \left(-\nu n^{2} \Lambda^{2} \tau^{3} / 3\right) .
\end{aligned}
$$

The asymptotic solutions for the scalar and the axial velocity are then given by (to order $\tau^{-1}$ )

$$
\begin{aligned}
& C(\rho, \theta, \tau)=C^{(0)}+\tau^{-1} \sum_{-\infty, n \neq 0}^{\infty} C_{n}^{(1)} \exp (i n(\theta-\Omega \tau)), \\
& C^{(0)}=\sum_{-\infty}^{\infty} C_{n}^{(0)}(\rho, \tau) \exp (i n(\theta-\Omega \tau)), \\
& C_{n}^{(0)}=\hat{C}_{n}^{(0)}(\rho) \exp \left(-D n^{2} \Lambda^{2} \tau^{3} / 3\right), \\
& C_{n}^{(1)}=\frac{i}{\rho} \sum_{-\infty, m \neq 0}^{\infty}\left(m \widetilde{\psi}_{m}^{(2)} \frac{\partial C_{n-m}^{(0)}}{\partial \rho}-(n-m) \frac{\partial \widetilde{\psi}_{m}^{(2)}}{\partial \rho} C_{n-m}^{(0)}\right), \\
& U_{3}(\rho, \theta, \tau)=U^{(0)}+\tau^{-1} \sum_{-\infty, n \neq 0}^{\infty} U_{n}^{(1)} \exp (i n(\theta-\Omega \tau)), \\
& U^{(0)}=\sum_{-\infty}^{\infty} U_{n}^{(0)}(\rho, \tau) \exp (i n(\theta-\Omega \tau)), \\
& U_{n}^{(0)}=\hat{U}_{n}^{(0)}(\rho) \exp \left(-\nu n^{2} \Lambda^{2} \tau^{3} / 3\right), \\
& U_{n}^{(1)}=\frac{i}{\rho} \sum_{-\infty, m \neq 0}^{\infty}\left(m \widetilde{\psi}_{m}^{(2)} \frac{\partial U_{n-m}^{(0)}}{\partial \rho}-(n-m) \frac{\partial \widetilde{\psi}_{m}^{(2)}}{\partial \rho} U_{n-m}^{(0)}\right),
\end{aligned}
$$

where the initial scalar field is given by the functions $\hat{c}_{n}^{(0)}(\rho)$ and the initial axial velocity field is given by the functions $\hat{U}_{n}^{(0)}(\rho)$. It should be emphasized that this scalar solution is only valid when the initial conditions have no $x_{3}$ dependence, as is the case for $c_{1}$ and $c_{2}$.

\section{THE CROSS SPECTRUM}

We will first consider the shell-summed cross spectrum defined by

$$
F_{u_{i}^{\prime} c}(k)=\frac{1}{(2 \pi)^{3}} \int_{S} \int_{V} R_{u_{i}^{\prime} c}\left(\mathbf{r}^{\prime}\right) e^{-i \mathbf{k}^{\prime} \cdot \mathbf{r}^{\prime}} d \mathbf{r}^{\prime} d \Omega_{k^{\prime}},
$$

where as before primed quantities indicate the lab frame, and of course $k=k^{\prime}$. The integral over $V$ indicates a volume integral that may be taken as over all space. The integral over $S$ indicates a surface integral over a spherical shell in wavenumber space. The shell-summed cross spectrum is real, as may be seen by making the change of integration variables $\mathbf{k}^{\prime}$ to $-\mathbf{k}^{\prime}$, and it integrates to the scalar flux,

$$
\overline{u_{i}^{\prime} c}=\int_{0}^{\infty} F_{u_{i}^{\prime} c}(k) d k
$$

We suppose there is a box populated by a collection of stretched vortex tubes. The vortex tubes do not interact except in that each vortex tube is stretched on average by the other vortex tubes. We assume that the vortex structures are distributed sparsely enough so that the overlapping velocity and scalar fields from the vortex tubes do not contribute strongly to the fine scales. It is further assumed that a statistical equilibrium has been reached whereby the structures are created and decay at the same rate. The average in the definition of the scalar-velocity correlation (1) is then interpreted as an average over time, space, vortex orientation, and initial conditions of one vortex tube,

$$
R_{u_{i}^{\prime} c}\left(\mathbf{r}^{\prime}\right)=N^{c}\left\langle\left\langle\int_{0}^{t_{c}} \int_{V} u_{i}^{\prime}\left(\mathbf{x}^{\prime}, t\right) c\left(\mathbf{x}^{\prime}+\mathbf{r}^{\prime}, t\right) d \mathbf{x}^{\prime} d t\right\rangle_{\mathrm{VO}}\right\rangle_{\mathrm{IC}},
$$

where $N^{c}$ is the rate of creation of vortex tubes per unit time and per unit volume, $t^{c}$ is a typical vortex lifetime, \langle\rangle$_{\text {IC }}$ indicates an average over initial conditions, and \langle\rangle$_{\mathrm{VO}}$ indicates an average over vortex orientation. The average over vortex orientation is defined using the Euler angles $\alpha, \beta, \gamma$ that rotate the lab frame to the vortex fixed frame, ${ }^{8}$

$$
\begin{aligned}
\left\langle f\left(E_{i j}\right)\right\rangle_{\mathrm{VO}}= & \frac{1}{8 \pi^{2}} \int_{0}^{2 \pi} \int_{0}^{2 \pi} \int_{0}^{\pi} f\left(E_{i j}\right) P(\alpha, \beta, \gamma) \\
& \times \sin \alpha d \alpha d \beta d \gamma,
\end{aligned}
$$

where $P(\alpha, \beta, \gamma)$ is the probability density function of the Euler angles. To match with experiment we specialize to the case of an isotropic velocity field and set $P=1$ so that all orientations are equally likely. We now rewrite Eq. (35) by changing the ordering of the averages, substituting $u_{i}^{\prime}$ $=E_{j i} u_{j}$ and changing integration variables from $\mathbf{r}^{\prime}$ to $\mathbf{r}$, from $\mathbf{k}^{\prime}$ to $\mathbf{k}$, and from $\mathbf{x}^{\prime}$ to $\mathbf{x}$, 


$$
\begin{aligned}
F_{u_{i}^{\prime} c}(k)= & \frac{N^{c}}{(2 \pi)^{3}}\left\langle\left\langle\int_{S} \int_{V} \int_{0}^{t_{c}} \int_{V} E_{j i} u_{j}(\mathbf{x}, t) c(\mathbf{x}+\mathbf{r}, t)\right.\right. \\
& \left.\left.\times e^{-i \mathbf{k} \cdot \mathbf{r}} d \mathbf{x} d t d \mathbf{r} d \Omega_{k}\right\rangle\right)
\end{aligned}
$$

\section{A. Simplification using symmetries in the distribution of initial conditions}

Next we use some symmetries in the distribution of the initial conditions of the velocity and the scalar to simplify Eq. (39). For a given vortex tube let the initial $u_{3}$ distribution be $u_{30}$ (say at the time when the tube is created). We will consider the effects of the transformation $u_{30}$ to $-u_{30}$. Clearly $u_{3}(\mathbf{x}, t)$ changes to $-u_{3}(\mathbf{x}, t)$ because Eq. (14) is linear and homogeneous. It is also clear that $u_{1}$ and $u_{2}$ are unaffected as they have no $x_{3}$ dependence. From Eq. (19) we see that initially $\partial c_{1} / \partial x_{3}=\partial c_{2} / \partial x_{3}=0$. Then by taking the partial derivative with respect to $x_{3}$ of Eq. (15) we see that $\partial c_{1} / \partial x_{3}=\partial c_{2} / \partial x_{3}=0$ for all times. It is then clear from Eq. (15) that $u_{3}$ has no influence on the evolution of $c_{1}$ or $c_{2}$. Finally, it is clear that the first term in expression (22) for $c_{3}$ is unaffected by changes in $u_{30}$, and that the second term will change sign when $u_{30}$ changes sign.

Therefore, if we assume that for each initial distribution of velocities $u_{1}(\mathbf{x}, 0)$ and $u_{2}(\mathbf{x}, 0)$ that $u_{3}(\mathbf{x}, 0)=u_{30}$ is as likely as $u_{3}(\mathbf{x}, 0)=-u_{30}$, then performing the average over the initial conditions will eliminate some terms that we now neglect. At this stage we also neglect the fluctuation term $c^{\prime}$ in the expression (18) for $c(\mathbf{x}, t)$. This may be justified by noting that $c^{\prime}(\mathbf{x}, t)$ depends linearly on $c^{\prime}(\mathbf{x}, 0)$, and making the approximation that $c^{\prime}(\mathbf{x}, 0)$ and $u_{j}(\mathbf{x}, 0)$ are statistically independent. We therefore replace $E_{j i} u_{j}(\mathbf{x}, t) c(\mathbf{x}+\mathbf{r}, t)$ in expression (39) with

$$
\begin{aligned}
& {\left[E_{1 i} u_{1}+E_{2 i} u_{2}\right](\mathbf{x}, t)\left[E_{11} c_{1}+E_{21} c_{2}\right.} \\
& \left.\quad+E_{31} \mu e^{-a t} x_{3}\right](\mathbf{x}+\mathbf{r}, t) \\
& \quad-E_{3 i} u_{3}(\mathbf{x}, t)\left[E_{31} \mu u_{3} \frac{1}{a} \sinh (a t)\right](\mathbf{x}+\mathbf{r}, t) .
\end{aligned}
$$

For simplicity we now replace the average over initial conditions with one particular initial condition. Using the integrals

$$
\frac{1}{8 \pi^{2}} \int E_{i j} E_{k 1} \sin \alpha d \alpha d \beta d \gamma=\frac{1}{3} \delta_{i k} \delta_{j 1}
$$

to perform the orientation average gives $F_{u_{2}^{\prime} c}=F_{u_{3}^{\prime} c}=0$ and

$$
\begin{aligned}
F_{u_{1}^{\prime} c}(k)= & \frac{1}{3(2 \pi)^{3}} \int_{S} \int_{V} \int_{0}^{t_{c}} \int_{V}\left[u_{1}(\mathbf{x}, t) c_{1}(\mathbf{x}+\mathbf{r}, t)+u_{2}(\mathbf{x}, t)\right. \\
& \left.\times c_{2}(\mathbf{x}+\mathbf{r}, t)-u_{3}(\mathbf{x}, t) \mu \frac{1}{a} \sinh (a t) u_{3}(\mathbf{x}+\mathbf{r}, t)\right] \\
& \times e^{-i \mathbf{k} \cdot \mathbf{r}} d \mathbf{x} d t d \mathbf{r} d \Omega_{k} .
\end{aligned}
$$

Noting that $u_{1}, u_{2}, u_{3}, c_{1}, c_{2}$ have no $x_{3}$ dependence we can replace $N^{c} \int_{0}^{t_{c}} \int_{V} d \mathbf{x} d t$ with $N \int_{0}^{t_{c}} \int_{-\infty}^{\infty} \int_{-\infty}^{\infty} d x_{1} d x_{2} S(t) d t$ where $N$ is the rate of creation of vortex length per unit time and per unit volume, and the factor $S(t)$ arises from the lengthening over time of the vortex tube. Defining the two dimensional Fourier transform of a function $f\left(x_{1}, x_{2}\right)$ by

$$
\begin{aligned}
\hat{f}\left(k_{1}, k_{2}\right)= & \frac{1}{4 \pi^{2}} \int_{-\infty}^{\infty} \int_{-\infty}^{\infty} e^{-i k_{1} x_{1}-i k_{2} x_{2}} \\
& \times f\left(x_{1}, x_{2}\right) d x_{1} d x_{2},
\end{aligned}
$$

and dividing the expression (42) into contributions from the axial velocity (a) and planar velocity (p), we find that

$$
F_{u_{1}^{\prime} c}(k)=F_{u_{1}^{\prime} c}^{(p)}(k)+F_{u_{1}^{\prime} c}^{(a)}(k)
$$

where

$F_{u_{1}^{\prime} c}^{(p)}(k)=\frac{N(2 \pi)^{2}}{3} \int_{0}^{t_{c}} \int_{0}^{2 \pi}\left(\hat{u}_{1} \hat{c}_{1}^{*}+\hat{u}_{2} \hat{c}_{2}^{*}\right) k d \theta_{k} S(t) d t$

$$
\begin{aligned}
F_{u_{1}^{\prime} c}^{(a)}(k)= & -\frac{N(2 \pi)^{2}}{3} \int_{0}^{t_{c}} \int_{0}^{2 \pi}\left(\mu \frac{1}{a} \sinh (a t) \hat{u}_{3} \hat{u}_{3}^{*}\right) \\
& \times k d \theta_{k} S(t) d t
\end{aligned}
$$

and $k_{1}=k \cos \theta_{k}$ and $k_{2}=k \sin \theta_{k}$.

\section{B. Contribution from the planar velocity: $F_{u_{1}^{\prime} c}^{(p)}$}

We now consider the contribution to the cross-spectrum from correlations between the planar velocities and $c_{1}$ and $c_{2}$ in Eq. (45). We can simplify the analysis by relating $\hat{\mathbf{u}}$ to $\hat{\omega}_{3}$. Using $\omega_{i}=\epsilon_{i j k} \partial u_{k} / \partial x_{j}$, assuming that the velocity field decays sufficiently quickly as $x_{1}$ or $x_{2}$ become large, and defining $k_{3}=0$ gives $\hat{\omega}_{l}=i \epsilon_{l m n} k_{m} \hat{u}_{n}$. The assumption of incompressibility gives $k_{l} \hat{u}_{l}=0$. Therefore $\hat{u}_{l}$ is orthogonal to $k_{l}$ and $\hat{\omega}_{l}$, and

$$
\hat{u}_{l}=\alpha \epsilon_{l m n} k_{m} \hat{\omega}_{n}=-\alpha i k^{2} \hat{u}_{l} .
$$

Thus the scalar $\alpha$ is determined and $\hat{u}_{l}\left(k_{1}, k_{2}\right)$ $=i k^{-2} \epsilon_{l m n} k_{m} \hat{\omega}_{n}$. We are interested in the components of the velocity in the plane,

$$
\hat{u}_{1}\left(k_{1}, k_{2}\right)=\frac{i}{k} \sin \theta_{k} \hat{\omega}_{3}, \quad \hat{u}_{2}\left(k_{1}, k_{2}\right)=-\frac{i}{k} \cos \theta_{k} \hat{\omega}_{3} .
$$

Then from Eq. (45) we have that

$$
\begin{aligned}
F_{u_{1}^{\prime} c}^{(p)}(k)= & \frac{1}{3} i N(2 \pi)^{2} \int_{0}^{t_{c}} \int_{0}^{2 \pi} \hat{\omega}_{3}\left(\sin \theta_{k} \hat{c}_{1}^{*}-\cos \theta_{k} \hat{c}_{2}^{*}\right) \\
& \times d \theta_{k} S(t) d t .
\end{aligned}
$$

Letting

$$
\omega_{3}=\sum_{n=-\infty}^{\infty} \omega_{n}(r, t) \exp (\text { in } \theta)
$$

and using

$$
\begin{gathered}
\int_{0}^{2 \pi} \exp \left(i n \theta-i k r \cos \left(\theta-\theta_{k}\right)\right) d \theta \\
=(-i)^{n} 2 \pi J_{n}(k r) \exp \left(i n \theta_{k}\right),
\end{gathered}
$$


gives

$$
\hat{\omega}_{3}=\frac{1}{2 \pi} \sum_{n=-\infty}^{\infty}(-i)^{n} \exp \left(\text { i n } \theta_{k}\right) I_{n}^{\omega}(k, t),
$$

where

$$
I_{n}^{\omega}(k, t)=\int_{0}^{\infty} \omega_{n}(r, t) J_{n}(k r) r d r .
$$

We will use the solution (33) for $c_{1}$ and $c_{2}$, but will neglect terms of order $\tau^{-1}$. Using the initial conditions (19) we see that for $c_{1}$

$$
\hat{C}_{1}^{(0)}=\frac{\mu \rho}{2}, \quad \hat{C}_{-1}^{(0)}=\frac{\mu \rho}{2}
$$

and that for $c_{2}$

$$
\hat{C}_{1}^{(0)}=-\frac{i \mu \rho}{2}, \quad \hat{C}_{-1}^{(0)}=\frac{i \mu \rho}{2} .
$$

Note that because the solutions for the scalar and the vorticity are only valid asymptotically in time, we should specify the initial conditions at some initial time $t_{1}>0$. However, we make the approximation $t_{1} \rightarrow 0$, and the resulting integrals are convergent at time zero.

It is convenient to define

$$
\begin{aligned}
I^{c}= & \frac{\mu}{2} \int_{0}^{\infty} \exp \left(-i \Omega(\rho) \tau-D \Lambda(\rho)^{2} \tau^{3} / 3\right) \\
& \times J_{1}(k r) \rho r d r \\
= & \frac{\mu}{2(1+a \tau)} \int_{0}^{\infty} \exp \left(-i \Omega(\rho) \tau-D \Lambda(\rho)^{2} \tau^{3} / 3\right) \\
& \times J_{1}\left(\frac{k \rho}{\sqrt{1+a \tau}}\right) \rho^{2} d \rho .
\end{aligned}
$$

Then using $J_{-1}=-J_{1}$ and Eq. (51) we have that

$$
\begin{aligned}
\hat{c}_{1}= & \frac{1}{2 \pi}\left((-i) \exp \left(i \theta_{k}\right) I^{c}+(-i)^{-1}\right. \\
& \left.\times \exp \left(-i \theta_{k}\right)\left(-I^{c}\right)^{*}\right), \\
\hat{c}_{2}= & \frac{1}{2 \pi}\left((-i) \exp \left(i \theta_{k}\right) I^{c}(-i)+(-i)^{-1}\right. \\
& \left.\times \exp \left(-i \theta_{k}\right)\left(-I^{c}\right)^{*} i\right) .
\end{aligned}
$$

Substituting Eqs. (57), (58), and (52) into (49), and after some algebra we find

$$
\begin{aligned}
F_{u_{1}^{\prime} c}^{(p)}(k)= & \frac{1}{3} N \int_{0}^{t_{c}} \int_{0}^{2 \pi} i \sum_{n=-\infty}^{\infty}(-i)^{n} \exp \left(i n \theta_{k}\right) \\
& \times I_{n}^{\omega}\left(\left(I^{c}\right)^{*}-I^{c}\right) d \theta_{k} S(t) d t
\end{aligned}
$$

Therefore only $n=0$ makes a contribution and

$$
F_{u_{1}^{\prime} c}^{(p)}(k)=\frac{4 \pi}{3} N \int_{0}^{t_{c}} I_{0}^{\omega} \operatorname{Im}\left(I^{c}\right) S(t) d t
$$

Finally we can evaluate $I_{0}^{\omega}$ in terms of $\Omega\left(r^{\prime}, 0\right)$ as follows:

$$
\begin{aligned}
I_{0}^{\omega} & =\int_{0}^{\infty} J_{0}(k r) \omega_{0}(r, t) r d r \\
& =\int_{0}^{\infty} J_{0}\left(\frac{k \rho}{\sqrt{1+a \tau}}\right) \widetilde{\omega}_{0}(\rho, \tau) \rho d \rho,
\end{aligned}
$$

where $\widetilde{\omega}_{0}(\rho, \tau)$ is the $\theta$ average of $\widetilde{\omega}_{3}(\rho, \theta, \tau)$. Note that unlike the analysis in Sec. II where $\Omega$ was taken as constant in time in the asymptotic solution for the scalar and vorticity spirals, we must now take into account the evolution in time of $\Omega\left(r^{\prime}, t\right)$ and $\widetilde{\omega}_{0}\left(r^{\prime}, t\right)$. It is easy to show that $\widetilde{\omega}_{0}$ evolves according to a heat equation in a cylindrical geometry. ${ }^{7}$ This can be solved using a Green's function in terms of the vorticity distribution at $t=0$,

$\widetilde{\omega}_{0}(\rho, \tau)=2 \pi \int_{0}^{\infty} \widetilde{\omega}_{0}\left(r^{\prime}, 0\right)$

$$
\times\left[\frac{1}{4 \pi \nu \tau} \exp \left(\frac{-\left(\rho^{2}+r^{\prime 2}\right)}{4 \nu \tau}\right) I_{0}\left(\frac{\rho r^{\prime}}{2 \nu \tau}\right)\right] r^{\prime} d r^{\prime}
$$

Substituting this into Eq. (61) and performing the $\rho$ integral gives

$$
I_{0}^{\omega}=\exp \left(\frac{-\nu \tau k^{2}}{1+a \tau}\right) \int_{0}^{\infty} J_{0}\left(\frac{r^{\prime} k}{\sqrt{1+a \tau}}\right) \widetilde{\omega}_{0}\left(r^{\prime}, 0\right) \quad r^{\prime} d r^{\prime} .
$$

If we use Eq. (31) to relate $\omega_{0}$ to $\Omega$ and note that $\omega_{0}$ and $\widetilde{\omega}_{0}$ coincide at $t=0$, we find that

$$
\begin{aligned}
I_{0}^{\omega}= & \exp \left(\frac{-\nu \tau k^{2}}{1+a \tau}\right) k(1+a \tau)^{-1 / 2} \\
& \times \int_{0}^{\infty} J_{1}\left(\frac{r^{\prime} k}{\sqrt{1+a \tau}}\right) \Omega\left(r^{\prime}, 0\right) r^{\prime 2} d r^{\prime} .
\end{aligned}
$$

This expression for $I_{0}^{\omega}$ cannot be evaluated using the method of stationary phase unlike the corresponding integrals for higher harmonics. Therefore $F_{u_{1}^{\prime} c}^{(p)}(k)$ does not have a universal form at high wave number, and in fact depends on the choice of $\Omega\left(r^{\prime}, 0\right)$. However, once again the contribution to the cross spectrum has no imaginary part. Combining Eqs. (60), (56) and (64) gives

$$
\begin{aligned}
F_{u_{1}^{\prime} c}^{(p)}(k)= & \frac{2 \pi \mu}{3} k N \int_{0}^{t_{c}} \exp \left(\frac{-\nu \tau k^{2}}{1+a \tau}\right)(1+a \tau)^{-3 / 2} \\
& \times S(t) L_{1}(\tau, k) L_{2}(\tau, k) d t,
\end{aligned}
$$

where

$$
\begin{aligned}
L_{1}(\tau, k)= & -\int_{0}^{\infty} J_{1}\left(\frac{k \rho}{\sqrt{1+a \tau}}\right) \sin (\Omega \tau) \\
& \times \exp \left(-D \Lambda^{2} \tau^{3} / 3\right) \rho^{2} d \rho, \\
L_{2}(\tau, k)= & \int_{0}^{\infty} J_{1}\left(\frac{r^{\prime} k}{\sqrt{1+a \tau}}\right) \Omega\left(r^{\prime}, 0\right) r^{\prime 2} d r^{\prime} .
\end{aligned}
$$


This expression is evaluated for a particular choice of vortex core in Sec. IV.

Unlike the calculation of $F_{u_{1}^{\prime} c}^{(a)}$ discussed in the next section, here we only calculate the lowest order contribution to $F_{u_{1}^{\prime} c}^{(p)}$. This is because of the complexity involved in proceeding to higher order, and also because, as we have seen, the asymptotic form of $F_{u_{1}^{\prime} c}^{(p)}$ is dependent on the initial conditions. We will use this lowest order contribution in Sec. IV to show that the planar contribution is comparable in magnitude to the axial contribution.

\section{Contribution from the axial velocity: $F_{u_{1}^{\prime} c}^{(a)}$}

We now wish to consider the contribution from correlations between the axial velocity $u_{3}$ and the second term of $c_{3}$. Using expression (46) and defining

$$
u_{3}(r, \theta, t)=\sum_{n=-\infty}^{\infty} u_{3, n}(r, t) \exp (i n \theta),
$$

we find that

$$
F_{u_{1}^{\prime} c}^{(a)}(k)=-\frac{\mu}{3 a} k N 2 \pi \int_{0}^{t_{c}} \sum_{n=-\infty}^{\infty}\left|I_{n}\right|^{2} \sinh (a t) S(t) d t,
$$

where

$$
I_{n}=\int_{0}^{\infty} J_{n}(k r) u_{3, n}(r, t) r d r .
$$

This is similar in structure to the expression for the energy spectrum contributed by the axial velocity. ${ }^{1}$ Note that this contribution is negative, consistent with the experimental results of Mydlarski and Warhaft. ${ }^{5}$ We can now use the asymptotic solution for $u_{3}$ to evaluate $I_{n}$. We first consider the contribution from the $U^{(0)}$ term. Using Eq. (34), the integral in $r$ for $I_{n}$ may be evaluated using the method of stationary phase giving

$\left|I_{n}\right|^{2}=\frac{\rho_{n}\left|\hat{U}_{n}^{(0)}\left(\rho_{n}\right)\right|^{2}}{S^{7 / 2} \Lambda^{\prime}\left(\rho_{n}\right) k \tau n} \exp \left(-\frac{2 \nu n^{2} \Lambda^{2}\left(\rho_{n}\right) \tau^{3}}{3}\right)$,

where $\rho_{n}$ is the point of stationary phase. If we approximate $S(t) \simeq a \tau$ (valid for $a \tau \gg 1$ ) then $\rho_{n}$ is related to $\tau$ by

$$
\tau \simeq\left(\frac{k}{n a^{1 / 2}\left|\Lambda\left(\rho_{n}\right)\right|}\right)^{2 / 3} .
$$

We now approximate $\sinh (a t)$ by $\frac{1}{2} \exp (a t)$ (making an $O\left(\tau^{-2}\right)$ error) and change integration variable from $t$ to $\rho$ $=\rho_{n}$. Using $\left|\hat{U}_{-n}^{(0)}\right|=\left|\hat{U}_{n}^{(0)}\right|$ and letting $t_{c} \rightarrow \infty$ we find

$$
\begin{aligned}
F_{u_{1}^{\prime} c}^{(a)}(k)^{(0)}= & -\frac{4 \pi \mu N}{9} k^{-5 / 3} a^{-8 / 3} \exp \left(-\frac{2 \nu k^{2}}{3 a}\right) \\
& \times \sum_{n=1}^{\infty} n^{2 / 3} \int_{0}^{\infty} \rho\left|\hat{U}_{n}^{(0)}(\rho)\right|^{2}|\Lambda(\rho)|^{2 / 3} d \rho,
\end{aligned}
$$

where we have neglected the zeroth harmonic.

\section{The next order contribution from $F_{u_{1}^{\prime} c}^{(a)}$}

Now consider the next order contribution by including terms involving $U_{n}^{(1)}$. We keep only terms $O\left(\tau^{-1}\right)$ and use stationary phase to evaluate the $r$ integral to find

$$
\begin{aligned}
\left|I_{n}\right|^{2}= & \frac{\rho_{n}\left(\hat{U}_{n}^{(0)}\left(\rho_{n}\right) U_{n}^{(1)}\left(\rho_{n}\right)^{*}+U_{n}^{(1)}\left(\rho_{n}\right) \hat{U}_{n}^{(0)}\left(\rho_{n}\right)^{*}\right)}{S^{7 / 2} \Lambda^{\prime}\left(\rho_{n}\right) k \tau^{2} n} \\
& \times \exp \left(-\frac{\nu n^{2} \Lambda^{2}\left(\rho_{n}\right) \tau^{3}}{3}\right) .
\end{aligned}
$$

Again approximating $\sinh (a t)$ by $\frac{1}{2} \exp (a t)$ for $a t \gg 1$, changing variables from $t$ to $\rho_{n}=\rho$, using $\left|\hat{U}_{-n}^{(0)}\right|=\left|\hat{U}_{n}^{(0)}\right|$ and letting $t_{c} \rightarrow \infty$ we find

$$
\begin{aligned}
F_{u_{1}^{\prime} c}^{(a)}(k)^{(1)}= & -\frac{8 \pi \mu N}{9} k^{-7 / 3} a^{-7 / 3} \exp \left(-\frac{\nu k^{2}}{3 a}\right) \\
& \times \sum_{n=1}^{\infty} n^{4 / 3} \int_{0}^{\infty}|\Lambda(\rho)|^{4 / 3} \operatorname{Re}\left(\hat{U}_{n}^{(0)}(\rho) P_{n}^{*}\right) d \rho,
\end{aligned}
$$

where

$$
\begin{aligned}
P_{n}= & i \sum_{m \neq 0} \exp \left(-\frac{\nu k^{2}\left(m^{2}+(n-m)^{2}\right)}{3 a n^{2}}\right) \\
& \times\left(\frac{f_{m}(\rho)}{m \Lambda^{2}} \frac{\partial}{\partial \rho}\left(\hat{U}_{n-m}^{(0)}(\rho)\right)\right. \\
& \left.-\frac{n-m}{m^{2}} \hat{U}_{n-m}^{(0)}(\rho) \frac{\partial}{\partial \rho}\left(\frac{f_{m}(\rho)}{\Lambda^{2}}\right)\right) .
\end{aligned}
$$

Again this contribution has no imaginary part and it is uniformly of one sign, although it is not clear if this sign is positive or negative. Note that the coefficient for this contribution is an integral involving the initial conditions for the axial velocity $\left(\hat{U}_{n}^{(0)}(\rho)\right)$, the axial vorticity $\left(f_{m}(\rho)\right)$ and the radial derivative of the theta averaged angular velocity $(\Lambda$ $=d \Omega / d \rho$ ). The following argument could be made to make this coefficient zero. The functions $f_{n}(r)$ describe the initial condition for the axial vorticity. We can write without loss of generality $f_{n}(r)=\exp \left(i n \theta_{n}+i n \delta\right)\left|f_{n}(r)\right|$ where the $\theta_{n}$ are constant offset angles and $\delta$ then fixes the orientation of the nonaxisymmetric part of the initial axial vorticity. Then $F_{u_{1}^{\prime} c}^{(a)}(k)^{(1)}$ only depends on $\delta$ through $P_{n}^{*}$. It is clear that if we assume $\delta$ is distributed uniformly for a given set of functions $\hat{U}_{n}^{(0)}(\rho)$ then $F_{u_{1}^{\prime} c}^{(a)}(k)^{(1)}$ will be zero. That is, if the initial conditions for the axial vorticity and the axial velocity are uncorrelated then this first order correction to $F_{u_{1}^{\prime} c}^{(a)}$ will give no contribution. Indeed changing the sign of the $f_{m}$ 's and leaving everything else constant will change the sign of this contribution.

Recall that earlier in the derivation we assumed that for a given initial condition for the velocity in the plane of the vortex, that either direction was as likely for the initial condition of the axial velocity. In other words it was assumed that the statistics of the initial velocity field do not possess 
chirality. This is consistent with the existence of a correlation between the orientation of the initial conditions of the axial velocity and the axial vorticity. Indeed changing the sign of $\hat{U}_{n}^{(0)}(\rho)$ has no effect on $F_{u_{1}^{\prime} c}^{(a)}(k)^{(1)}$.

In summary, we have found an expression for the axial contribution as a sum of two terms. The first term, expression (73), has a $k^{-5 / 3}$ power law range. This is produced by the winding of the initial axial velocity field by the axisymmetric vortex core. The next order term, expression (75), has a $k^{-7 / 3}$ power law range, and arises from the lowest order effect of the nonaxisymmetric vorticity on the evolution of the axial velocity. We will evaluate Eqs. (73) and (75) in Sec. IV for a particular choice of initial conditions.

\section{COMPARISON WITH EXPERIMENT}

We now evaluate Eqs. (65), (73) and (75) for a particular choice of initial conditions. For simplicity and consistency we will choose simple initial conditions similar to those considered for the scalar spectrum by PL. We will use a line vortex as the initial condition for the vortex core so that

$$
\Omega(\rho, 0)=\frac{\Gamma}{2 \pi \rho^{2}}, \quad \Lambda(\rho, 0)=-\frac{\Gamma}{\pi \rho^{3}} .
$$

\section{A. Contribution of the axial velocity}

We first consider the contribution of the axial velocity. The initial condition for the non-axisymmetric component of the axial vorticity is chosen to be

$$
\omega_{3}(r, \theta, 0)=2 f_{0} g(\rho) \sin (2 \theta),
$$

so that $f_{2}=-i f_{0} g$ and $f_{-2}=i f_{0} g$ where $f_{0}$ is a dimensional constant. We also assume the initial condition for the axial velocity to be

$$
U_{3}(r, \theta, 0)=2 u_{0} g(\rho) \cos \theta
$$

so that $\hat{U}_{1}^{(0)}=u_{0} g$ and $\hat{U}_{-1}^{(0)}=u_{0} g$. Then

$$
\begin{aligned}
F_{u_{1}^{\prime} c}^{(a)}(k)= & -\frac{4 \pi \mu N}{9} k^{-5 / 3} a^{-8 / 3} \exp \left(-\frac{2 \nu k^{2}}{3 a}\right) u_{0}^{2} A_{0} \\
& -\frac{8 \pi \mu N}{9} k^{-7 / 3} a^{-7 / 3} \exp \left(-\frac{2 \nu k^{2}}{a}\right) f_{0} u_{0}^{2} B_{0},
\end{aligned}
$$

where

$$
\begin{aligned}
& A_{0}=\int_{0}^{\infty} \rho g(\rho)^{2}|\Lambda(\rho)|^{2 / 3} d \rho, \\
& B_{0}=\int_{0}^{\infty}|\Lambda(\rho)|^{4 / 3}\left(\frac{g^{2}}{2 \Lambda^{2}} \frac{d g}{d \rho}+\frac{g^{2}}{4} \frac{d}{d \rho}\left(\frac{g}{\Lambda^{2}}\right)\right) d \rho .
\end{aligned}
$$

We now choose a simple form for $g(\rho)$. Letting $R$ be a characteristic vortex radius we set $g(\rho)=1$ for $R / 2<\rho<R$, and zero otherwise. Note that the cutoffs in $g(\rho)$ do not create a spurious contribution at high wave number to the form of the cross spectrum as we only use $g(\rho)$ to evaluate coefficients for the axial velocity contribution. Then $A_{0}$ and $B_{0}$ may be evaluated, taking care to deal with the derivatives at the cutoffs in $g(\rho)$,

$$
A_{0}=\left(\frac{\Gamma}{\pi}\right)^{2 / 3} \log 2, \quad B_{0}=\frac{3 \pi^{2 / 3}}{8} \frac{R^{2}}{\Gamma^{2 / 3}} .
$$

We next approximate the strain rate using $a=(\epsilon /(15 \nu))^{1 / 2}$ and choose $f_{0}=\Gamma / R^{2}, u_{0}=\Gamma / R$. Then we can write

$$
\begin{aligned}
\frac{F_{u_{1}^{\prime} c}^{(a)}(k)}{\mu \epsilon^{-1 / 4} \nu^{7 / 4}} & \\
= & -\frac{1}{36} \log 2 \pi^{1 / 3} 15^{-1 / 6}(k \eta)^{-5 / 3} \\
& \times \exp \left(-2.58(k \eta)^{2}\right)\left(\frac{N R^{2}}{a}\right)\left(\frac{\Gamma}{\nu}\right)^{8 / 3}\left(\frac{a R^{2}}{4 \nu}\right)^{-2} \\
& -\frac{1}{48} \pi^{5 / 3} 15^{-1 / 3}(k \eta)^{-7 / 3} \\
& \times \exp \left(-7.75(k \eta)^{2}\right)\left(\frac{N R^{2}}{a}\right)\left(\frac{\Gamma}{\nu}\right)^{7 / 3}\left(\frac{a R^{2}}{4 \nu}\right)^{-2},
\end{aligned}
$$

where $\eta$ is the Kolmogorov length scale. It is interesting to find the ratio of the two terms in the above expression,

$$
(k \eta)^{-2 / 3} \frac{3 \pi^{4 / 3}}{4 \log 2} 15^{-1 / 6}\left(\frac{\Gamma}{\nu}\right)^{-1 / 3} \exp \left(-5.17(k \eta)^{2}\right) .
$$

Thus the second term becomes less important as the vortex Reynold's number increases.

To compare this with the experiment of Mydlarski and Warhaft ${ }^{5}$ it is necessary to estimate $N R^{2} / a, \Gamma / \nu$ and $a R^{2} /(4 \nu)$. We assume a value of $\Gamma / \nu=1000$ and let $R$ be given by the Taylor length scale. All other parameters except $N$ are taken from the table of parameters in Mydlarksi and Warhaft's paper ${ }^{5}$ for a Taylor Reynolds number of 582.

To estimate $N$ we find an expression involving $N$ for the energy dissipation from the model and compare it with the experimental value. The energy dissipation for the model is given by

$$
\epsilon=3 \nu a^{2}+2 \nu \int_{0}^{\infty} k^{2}\left(E_{0}(k)+E_{s}(k)+E_{\omega_{\theta}}\right) d k .
$$

The first term is the dissipation from the strain field. The remaining three terms are the dissipation associated with the vortex core, (axial) vortex spiral and axial velocity, respectively. This is Eq. (37) in Pullin and Saffman, ${ }^{7}$ except that we now also include the leading order dissipation from the axial velocity. An expression for $E_{\omega_{\theta}}$ is given by Eq. (66) of PL. Expressions for $E_{s}$ and $E_{0}$ are given in Pullin and Saffman, ${ }^{7}$ and were evaluated for the current choice of initial conditions. The core dissipation was found to be

$$
2 \nu \int_{0}^{\infty} k^{2} E_{0} d k=\frac{\Gamma^{2} N}{8 \pi} \int_{\tau_{1}}^{\tau_{2}} \frac{1+a \tau}{\tau} d \tau .
$$

To obtain a finite value we must choose reasonable time cutoffs $\tau_{1}$ and $\tau_{2}$, and, following Pullin et al. ${ }^{10}$ we choose $a \tau_{1}=1$ and $\tau_{2}=(\Gamma / \nu)^{-2 / 3} 10 R^{2} /(4 \nu)$. 


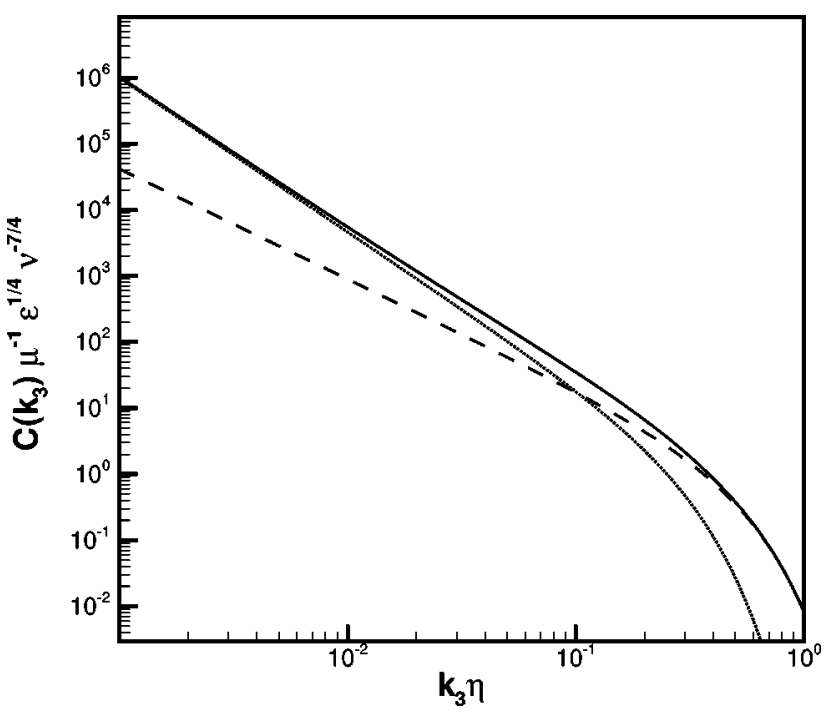

FIG. 1. The $1 \mathrm{D}$ cospectrum contributed by the axial velocity. Dashed, $-5 / 3$ component. Dotted, $-7 / 3$ component. Solid, both components.

In order to make comparisons with the data of Mydlarski and Warhaft ${ }^{5}$ it is necessary to convert our shell-summed cross spectrum to a one-dimensional cospectrum. The appropriate relationship is derived in Appendix A,

$$
C_{u_{1}^{\prime} c}^{1 d}\left(k_{3}^{\prime}\right)=\frac{3}{4} \int_{k_{3}^{\prime}}^{\infty} \frac{k^{2}+k_{3}^{\prime 2}}{k^{3}} F_{u_{1}^{\prime} c}(k) d k .
$$

We note the factor of 2 in the relationship between the onedimensional cross spectrum and cospectrum.

It is interesting to observe that if the one-dimensional cospectrum had been measured in the same direction as the velocity component used $\left(u_{1}^{\prime}\right)$ [i.e., if $C_{u_{1}^{\prime} c}^{1 d}\left(k_{1}^{\prime}\right)$ had been measured], then the axial velocity would give no contribution because the axial velocity does not generate small scale scalar structure in the axial direction. The normalized onedimensional cospectrum contributed by the axial velocity, obtained from Eqs. (84) and (88), is shown in Fig. 1 where the $k^{-5 / 3}$ and the $k^{-7 / 3}$ terms are also shown separately for comparison. In Fig. 2 this contribution to the cospectrum is compared with the experimental result of Mydlarski and Warhaft. Note that the exact magnitude of this contribution is dependent on several rough estimates made above, for example the value chosen for $\tau_{2}$. In Fig. 3 the same comparison is made, except now the experimental data has been smoothed using a one-third octave filter. The same comparison is again shown in Fig. 4 in compensated form, where the spectra have been multiplied by $\left(k_{3}^{\prime} \eta\right)^{2}$.

\section{B. Contribution of the planar velocity}

Equation (65) for the contribution to the cross spectrum of the velocity in the plane of the vortex cannot in general be evaluated analytically or asymptotically. It was instead evaluated numerically for different choices of the initial condition for the vortex core. A combination of the monte-carlo integration routine "Vegas" 11 and the double exponential routine of Ooura ${ }^{12}$ was used. The double exponential routine

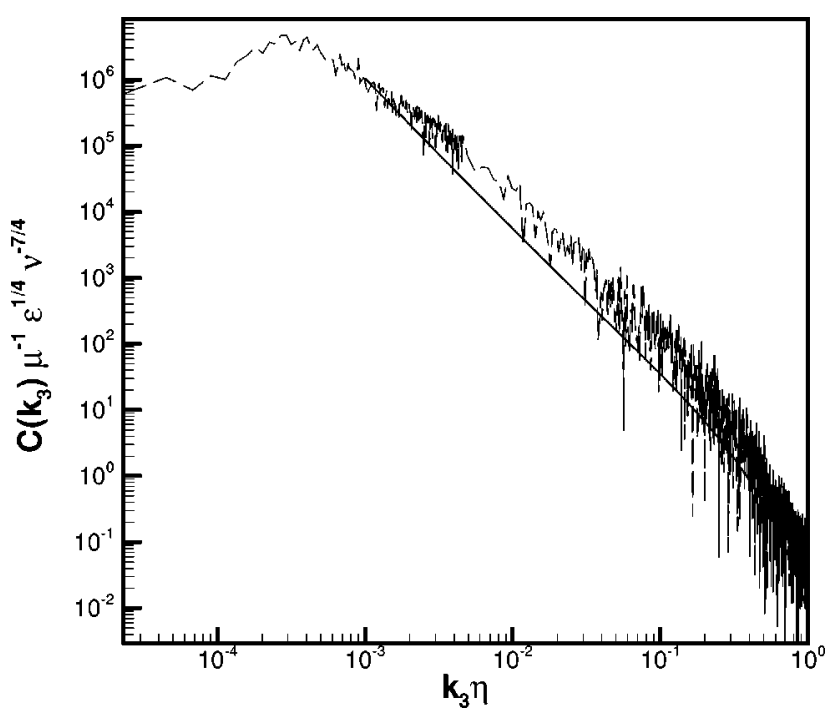

FIG. 2. The 1D cospectrum contributed by the axial velocity compared with experiment. Dashed, Mylardski and Warhaft data (Ref. 5). Solid, axial contribution from model.

was used to speed convergence of the oscillatory $I^{c}$ integral for large $\rho$. This method of numerical integration was first used to evaluate the energy spectrum and reasonable agreement with Lundgren's asymptotic result ${ }^{6}$ was found. In the case of an initial condition for the vortex core of a line vortex (77) the situation is simplified because the $I_{0}^{\omega}$ integral can be performed explicitly. The resulting contribution to the one-dimensional cospectrum for this initial condition is shown on a log-log scale in Fig. 5 compared with the axial contribution.

Note that the planar contribution is not of one sign, unlike the experimental result. This is typical of results obtained for other choices of the initial condition for the vortex core. Also the upper cutoff in time has an effect on this contribution, and if a cutoff is not used the core winds up

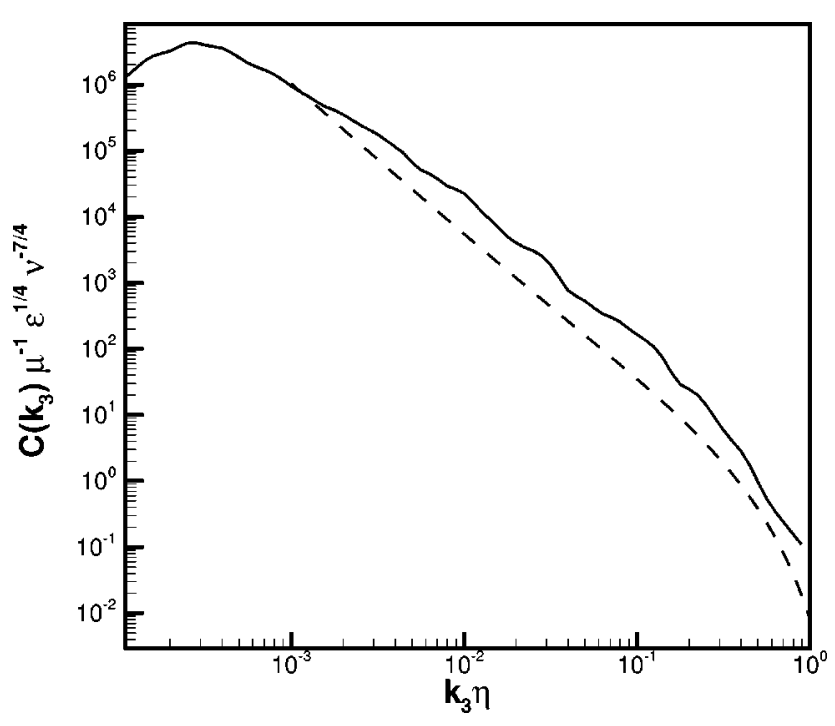

FIG. 3. The 1D cospectrum contributed by the axial velocity compared with the 1/3 octave filtered experimental data. Dashed, axial contribution from model. Solid, 1/3 octave filtered Mydlarski and Warhaft data (Ref. 5). 


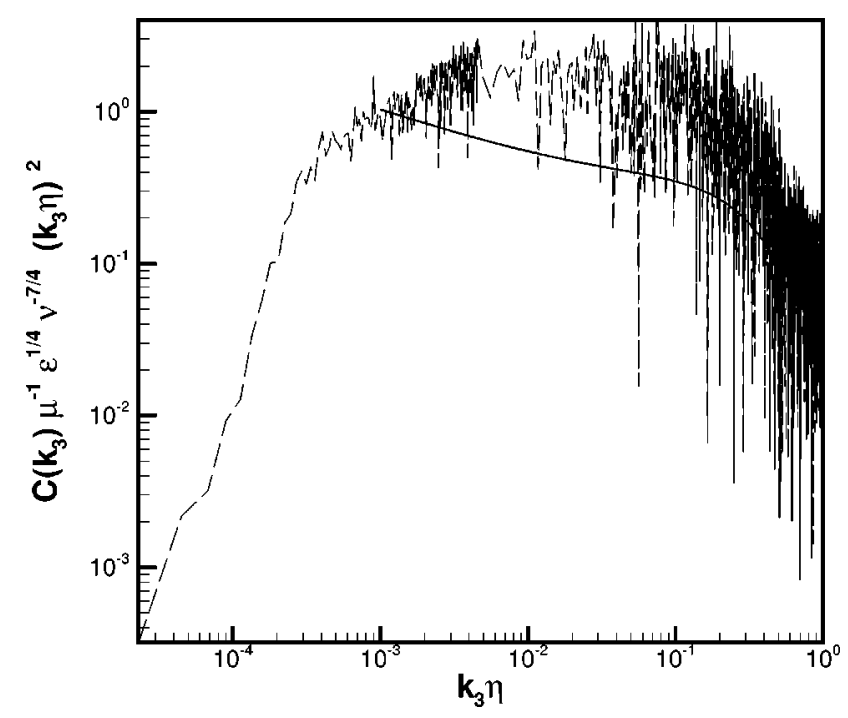

FIG. 4. The 1D cospectrum contributed by the axial velocity compared with experiment in compensated form. Solid, axial contribution from model. Dashed, Mydlarski and Warhaft data (Ref. 5).

more and more of the gradient so that the integrals do not converge. The planar contribution for this initial condition has two distinct ranges. The first range (for low wave number) is a $k^{-1}$ range and is of one sign. At higher wave number there is an oscillatory spectrum with a $k^{-5 / 3}$ envelope. However, both of these ranges are specific to this choice of $\Omega$ because the integral in $I_{0}^{\omega}$ is not in general dominated by a point of stationary phase, and its form varies for different $\Omega$ 's.

\section{CONCLUSION}

The stretched vortex model has been used to calculate the velocity-scalar cross spectrum for isotropic turbulence in the presence of an imposed mean scalar gradient. The

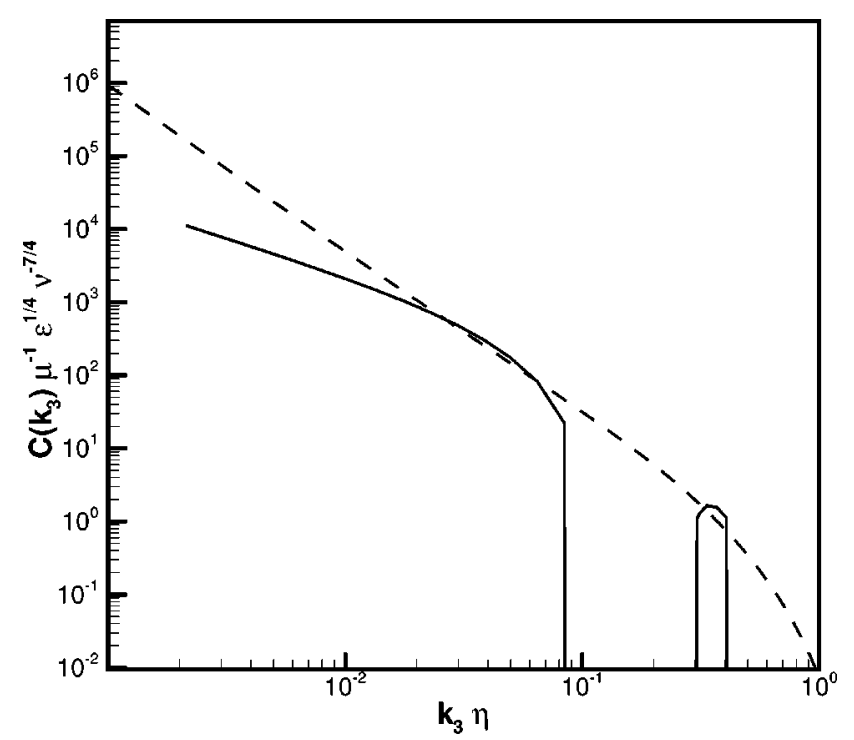

FIG. 5. Comparison of the 1D cospectrum contributed by the axial velocity and the planar velocity. Solid, planar contribution. Dashed, axial contribution. quadrature spectrum was found to be zero in agreement with experiment. Asymptotic expressions were found for the cospectrum contributed by the axial velocity of the vortex, with the leading order term showing a $k^{-5 / 3}$ range. The next order term had a $k^{-7 / 3}$ range, but its coefficient could be of either sign or zero. It is interesting that Mydlarski and Warhaft ${ }^{5}$ find a spectral exponent close to -2 , which lies in between those of the leading-order terms contributed by the axial velocity in the present model. The contribution to the cospectrum from the velocity in the plane of the vortex was also calculated, but some integrals had to be evaluated numerically, and a specific vortex core had to be chosen. The resulting contribution did not remain of one sign, and was comparable in magnitude to the axial contribution. The form of the contribution from the planar velocity depended on the choice of vortex core and time cutoff, and so a more physical contribution to the cospectrum might result from an ensemble of different vortex cores and lifetimes.

\section{ACKNOWLEDGMENTS}

We wish to thank L. Mydlardski for providing experimental data, and also P. Dimotakis for useful suggestions. P.A.O'G. and D.I.P. were supported in part by the National Science Foundation under Grant No. CTS-9978551.

\section{APPENDIX A: PROPERTIES OF THE VELOCITY-SCALAR CROSS SPECTRUM FOR ISOTROPIC TURBULENCE WITH A MEAN SCALAR GRADIENT}

The velocity field is assumed to be statistically isotropic and homogeneous. The scalar field is statistically homogeneous with a mean scalar gradient given by the vector $\mu_{i}$. We define $\mathcal{F}_{i}(\mathbf{k})$ to be the Fourier transform of $R_{u_{i} c}(\mathbf{r})$,

$$
\mathcal{F}_{i}(\mathbf{k})=\frac{1}{(2 \pi)^{3}} \int R_{u_{i} c}(\mathbf{r}) e^{-i \mathbf{k} \cdot \mathbf{r}} d \mathbf{r} .
$$

Then $\mathcal{F}_{i}$ is a first order tensor that is axisymmetric about $\mu_{i}$, and so must have the following form:

$$
\mathcal{F}_{i}(\mathbf{k})=A k_{i}+B \mu_{i},
$$

where $A$ and $B$ are functions of $k_{i} \mu_{i}, k$ and $\mu$. Using the incompressibility of the velocity field we have that

$$
k_{i} \mathcal{F}_{i}=A k^{2}+B \mu_{i} k_{i}=0 .
$$

Using this relation, and defining $D=-A k^{2} /\left(\mu_{j} k_{j}\right)$, we find that

$$
\mathcal{F}_{i}(\mathbf{k})=D\left(k, \mu, k_{j} \mu_{j}\right)\left(\mu_{i}-\frac{k_{j} \mu_{j}}{k^{2}} k_{i}\right) .
$$

We will now use the fact that the equation governing the scalar, Eq. (8), is linear. If the spectrum is measured after a sufficiently long time, the effect of initial fluctuations about the mean gradient will have decayed to zero, and the scalar must have a linear dependence on the vector $\mu_{i}$. Also $\mathcal{F}_{i}(\mathbf{k})$ depends linearly on the scalar, and so must also depend linearly on $\mu_{i}$. Therefore $D=D(k)$ is a function of $k$, and has no dependence on $k_{i} \mu_{i}$ or $\mu$. A similar analysis to the above is given in Herr, Wang and Collins. ${ }^{9}$ 
We will now use this simplified form for $\mathcal{F}_{i}(\mathbf{k})$ to relate the shell-summed and one-dimensional cross spectra. We specialize to the case where $\mu_{1}=\mu$ and $\mu_{2}=\mu_{3}=0$, and consider the one-dimensional cross spectrum in the $k_{3}$ direction. Then it is easy to show that

$$
F_{u_{i} c}^{1 d}\left(k_{3}\right)=\int_{-\infty}^{\infty} \int_{-\infty}^{\infty} \mathcal{F}_{i}(\mathbf{k}) d k_{1} d k_{2} .
$$

Changing to polar coordinates $k_{1}=\kappa \cos \theta$ and $k_{2}=\kappa \sin \theta$, and performing the $\theta$ integral gives $F_{u_{2} c}^{1 d}\left(k_{3}\right)=F_{u_{3} c}^{1 d}\left(k_{3}\right)=0$. Making a further change of integration variables from $\kappa$ to $k$ gives

$$
F_{u_{i} c}^{1 d}\left(k_{3}\right)=\mu \pi \int_{k_{3}}^{\infty} D(k)\left(k+\frac{k_{3}^{2}}{k}\right) d k .
$$

We can also find the shell-summed cross spectrum in terms of $D(k)$ as follows:

$$
F_{u_{i} c}(k)=\int_{S} \mathcal{F}_{i}(\mathbf{k}) d \Omega_{k} .
$$

Using spherical polar coordinates we find that $F_{u_{2} c}(k)$ $=F_{u_{3} c}(k)=0$, and

$$
F_{u_{1} c}(k)=\frac{8 \pi}{3} k^{2} D(k) \mu .
$$

Then comparing Eqs. (A6) and (A8) we have that

$$
F_{u_{1} c}^{1 d}\left(k_{3}\right)=\frac{3}{8} \int_{k_{3}}^{\infty} \frac{k^{2}+k_{3}^{2}}{k^{3}} F_{u_{1} c}(k) d k
$$

Finally we can easily show that the quadrature spectrum, $Q_{u_{1} c}^{1 d}\left(k_{3}\right)$, must be zero. Noting that $\mathcal{F}_{i}(\mathbf{k})$ is the Fourier transform of a real function gives $\mathcal{F}_{i}(-\mathbf{k})=\mathcal{F}_{i}^{*}(\mathbf{k})$. Also Eq. (A4) implies that $\mathcal{F}_{i}(-\mathbf{k})=\mathcal{F}_{i}(\mathbf{k})$, and so $D(k)$ is real, and the quadrature spectrum must be zero.

\section{APPENDIX B: CONTRIBUTION TO THE SCALAR SPECTRUM FROM $c_{3}$}

In their calculation of the scalar spectrum PL assumed that the scalar had no $x_{3}$ dependence. We will now consider the effect of an $x_{3}$ dependence in the particular case when the scalar initial condition is given by a gradient in the lab frame. An expression for the scalar spectrum is given by replacing $E_{j i} u_{j}(\mathbf{x}, t)$ with $c(\mathbf{x}, t)$ in Eq. (39),

$$
\begin{aligned}
E_{c}(k)= & \frac{N^{c}}{(2 \pi)^{3}}\left\langle/ \int_{S} \int_{V} \int_{0}^{t_{c}} \int_{V} c(\mathbf{x}, t) c(\mathbf{x}+\mathbf{r}, t)\right. \\
& \left.\left.\times e^{-i \mathbf{k} \cdot \mathbf{r}} d \mathbf{x} d t d \mathbf{r} d \Omega_{k}\right\rangle_{\mathrm{VO}}\right\rangle_{\mathrm{IC}}
\end{aligned}
$$

We decompose $c(\mathbf{x}, t)$ using Eq. (18), and assume the initial conditions (19). We set $c^{\prime}(\mathbf{x}, 0)=0$, because our solution (22) for $c_{3}$ is only valid for a gradient initial condition. This approximation was not necessary in the case of the cross spectrum, where we were able to eliminate the $c^{\prime}$ contribution by making an assumption about the statistical distribution of initial conditions. The orientation average may be performed immediately using the integrals (41) to give

$$
\begin{aligned}
E_{c}(k)= & \frac{N^{c}}{3(2 \pi)^{3}}\left\langle\int_{S} \int_{V} \int_{0}^{t_{c}} \int_{V} \sum_{j=1}^{3} c_{j}(\mathbf{x}, t) c_{j}(\mathbf{x}+\mathbf{r}, t)\right. \\
& \left.\times e^{-i \mathbf{k} \cdot \mathbf{r}} d \mathbf{x} d t d \mathbf{r} d \Omega_{k}\right\rangle_{\mathrm{IC}}
\end{aligned}
$$

The terms in the integrand involving $c_{1}$ and $c_{2}$ have no $x_{3}$ dependence and so were dealt with in PL. We will consider $E_{c_{3}}$, the contribution from $c_{3}$. We assume $\nu=D$ and use solution (22) for $c_{3}$. Then we have that

$$
\begin{aligned}
c_{3}(\mathbf{x}, t) c_{3}(\mathbf{x}+\mathbf{r}, t)= & \mu^{2} \exp (-2 a t)\left(x_{3} r_{3}+x_{3}^{2}\right)-\mu^{2} a^{-1} \\
& \times \exp (-a t) \sinh (a t)\left(x_{3} u_{3}(\mathbf{x}+\mathbf{r}, t)\right. \\
& \left.+x_{3} u_{3}(\mathbf{x}, t)+r_{3} u_{3}(\mathbf{x}, t)\right)+\mu^{2} a^{-2} \\
& \times \sinh ^{2}(a t) u_{3}(\mathbf{x}, t) u_{3}(\mathbf{x}+\mathbf{r}, t) .
\end{aligned}
$$

We will now show that the first term in Eq. (22) does not contribute to the scalar spectrum. Noting that $u_{3}(\mathbf{x})$ has no $x_{3}$ dependence, terms in Eq. (B3) that are linear in $x_{3}$ will be eliminated upon integration over $x_{3}$. Also terms that are linear in $u_{3}$ will be eliminated by performing the average over initial conditions. The term involving $x_{3}^{2}$ will not contribute to the high wave number spectrum. Therefore the only contribution comes from the second term of Eq. (22). We again simplify by replacing the average over initial conditions with one particular initial condition.

Changing to Fourier space using Eq. (43), and after some algebra, we find

$$
\begin{aligned}
E_{c_{3}}(k)= & \frac{N(2 \pi)^{2}}{3} \int_{0}^{t_{c}} \int_{0}^{2 \pi} \mu^{2} a^{-2} \\
& \times \sinh ^{2}(a t) \hat{u}_{3} \hat{u}_{3}^{*} k d \theta_{k} S(t) d t .
\end{aligned}
$$

This is very similar to expression (46) for the axial contribution to the cross spectrum, except for a factor in the integrand of $-\mu / a \sinh (a t)$. Following a similar analysis to Secs. III C and III D, and keeping terms in the integrand of $O\left(\tau^{-1}\right)$, we find

$$
\begin{aligned}
E_{c_{3}}(k)= & \frac{2 \pi \mu^{2} N}{9} k^{-1} a^{-3} \exp \left(-\frac{2 \nu k^{2}}{3 a}\right) \\
& \times \sum_{n=1}^{\infty} \int_{0}^{\infty} \rho\left|\hat{U}_{n}^{(0)}(\rho)\right|^{2} d \rho \\
& +\frac{4 \pi \mu^{2} N}{9} k^{-5 / 3} a^{-8 / 3} \exp \left(-\frac{\nu k^{2}}{3 a}\right) \\
& \times \sum_{n=1}^{\infty} n^{2 / 3} \int_{0}^{\infty}|\Lambda(\rho)|^{2 / 3} \operatorname{Re}\left(\hat{U}_{n}^{(0)}(\rho) P_{n}^{*}\right) d \rho .
\end{aligned}
$$

Thus the new contribution to $E_{c}$ is a combination of $k^{-1}$ and $k^{-5 / 3}$ power laws in a certain range. The wave number 
dependence is the same as was found in PL, Eqs. (81)-(85), for scalar initial conditions with no $x_{3}$ dependence, and so the new contribution does not alter the nature of the spectrum.

${ }^{1}$ D.I. Pullin and T.S. Lundgren, "Axial motion and scalar transport in stretched spiral vortices," Phys. Fluids 13, 2553 (2001).

${ }^{2}$ D.I. Pullin, "A vortex-based model for the subgrid flux of a passive scalar," Phys. Fluids 12, 2311 (2000).

${ }^{3}$ J. Bendat and A. Piersol, Random Data-Analysis and Measurement Procedures, 2nd ed. (Wiley, New York, 1986).

${ }^{4}$ J.L. Lumley, "Similarity and the turbulent energy spectrum," Phys. Fluids 10, 855 (1967).

${ }^{5}$ L. Mydlarski and Z. Warhaft, "Passive scalar statistics in high-Pécletnumber grid turbulence,” J. Fluid Mech. 358, 135 (1998).
${ }^{6}$ T.S. Lundgren, "Strained spiral vortex model for turbulent fine structure," Phys. Fluids 25, 2193 (1982).

${ }^{7}$ D.I. Pullin and P.G. Saffman, "On the Lundgren-Townsend model of turbulent fine scales," Phys. Fluids A 5, 126 (1993).

${ }^{8}$ D.I. Pullin and P.G. Saffman, "Reynolds stresses and one-dimensional spectra for a vortex model of homogeneous anisotropic turbulence," Phys. Fluids 6, 1787 (1994).

${ }^{9}$ S. Herr, L. Wang, and L.R. Collins, "EDQNM model of a passive scalar with a uniform mean gradient," Phys. Fluids 8, 1588 (1996).

${ }^{10}$ D.I. Pullin, J.D. Buntine, and P.G. Saffman, "On the spectrum of a stretched spiral vortex," Phys. Fluids 6, 3010 (1994).

${ }^{11}$ W.H. Press, S.A. Teukolsky, W.T. Vetterling, and B.P. Flannery, Numerical Recipes in $C$, 2nd ed. (Cambridge University Press, Cambridge, MA, 1992).

${ }^{12} \mathrm{~T}$. Ooura and M. Mori, “The double exponential formula for oscillatory functions over the half infinite interval," J. Comput. Appl. Math. 38, 353 (1991). 